\title{
Brief Intraperitoneal Radioimmunotherapy of Small Peritoneal Carcinomatosis Using High Activities of Noninternalizing ${ }^{125}$ I-Labeled Monoclonal Antibodies
}

\author{
Vincent Boudousq ${ }^{1-5}$, Stéphanie Ricaud ${ }^{1-4}$, Véronique Garambois ${ }^{1-4}$, Caroline Bascoul-Mollevi ${ }^{4}$, Samir Boutaleb ${ }^{1-4}$, \\ Muriel Busson ${ }^{1-4}$, François Quenet ${ }^{4}$, Pierre-Emmanuel Colombo ${ }^{4}$, Manuel Bardiès ${ }^{6}$, Pierre-Olivier Kotzki ${ }^{1-5}$, \\ Isabelle Navarro-Teulon ${ }^{1-4}$, André Pèlegrin ${ }^{1-4}$, and Jean-Pierre Pouget ${ }^{1-4,7}$ \\ ${ }^{1}$ IRCM, Institut de Recherche en Cancérologie de Montpellier, Montpellier, France; ${ }^{2}$ INSERM, U896, Montpellier, France; \\ ${ }^{3}$ Université Montpellier 1, Montpellier, France; ${ }^{4}$ CRLC Val d'Aurelle Paul Lamarque, Montpellier, France; ${ }^{5}$ Service de Médecine \\ Nucléaire, CHU de Nîmes, Nîmes, France; ${ }^{6} I N S E R M$, U892, Nantes, France; and ${ }^{7}$ Direction de la Radioprotection de l'Homme, \\ Institut de Radioprotection et de Sûreté Nucléaire, Fontenay-aux-Roses, France
}

We assessed the efficiency and toxicity of brief intraperitoneal radioimmunotherapy using high activities of ${ }^{125}$ l-labeled monoclonal antibody (mAb) in the treatment of small-volume peritoneal carcinomatosis. Methods: Brief intraperitoneal radioimmunotherapy consisted of a $185-\mathrm{MBq}(740 \mathrm{MBq} / \mathrm{mg})$ intraperitoneal injection of ${ }^{125} \mathrm{I}-35 \mathrm{~A} 7$ (an anti-carcinoembryonic antigen $\mathrm{mAb}$ ) into athymic nude mice $4 \mathrm{~d}$ after peritoneal tumor xenografting and, after $1 \mathrm{~h}$, abundant washing of the peritoneal cavity with saline solution to remove unbound radioactivity. Another group of mice received this treatment plus a $37-\mathrm{MBq}$ intravenous injection of ${ }^{125} \mathrm{I}-35 \mathrm{~A} 7$ on day 7 or 11 after grafting. Control groups received a brief treatment followed by an additional intravenous injection on day 7 of either saline solution or irrelevant ${ }^{125} \mathrm{I}-\mathrm{PX}$. Tumor growth was monitored by bioluminescence imaging and SPECT/CT, and hematologic toxicity was evaluated by complete blood counts. Survival time was reported, and the mice were sacrificed when the bioluminescence signal reached $4.5 \times 10^{7}$ photons/s. The biodistribution of 125I-35A7 mAb after intravenous or brief treatment was assessed, and the mean absorbed irradiation dose by organs and tumors was calculated using the MIRD formalism. Results: Mild, transient hematologic toxicity was observed after the brief treatment plus intravenous ${ }^{125} \mathrm{I}-\mathrm{mAb}$, with no weight loss. Median survival increased from $32 \mathrm{~d}$ in the control groups, to $46 \mathrm{~d}$ in the brief treatment group, to $66 \mathrm{~d}$ in the group additionally receiving intravenous treatment on day 11 , to $73 \mathrm{~d}$ in the group additionally receiving intravenous treatment on day 7 . The brief treatment alone resulted in a 3-fold higher tumor-toblood uptake ratio than did the standard intravenous treatment, and the mean absorbed irradiation doses by tumors were 11.6 Gy for the brief treatment and 16.7 Gy for the additional intravenous treatment. For healthy tissues other than blood, the mean absorbed irradiation dose did not exceed 1 Gy after brief treatment and 4.2 Gy after intravenous treatment. Conclusion: The efficiency, low toxicity, and high tumor-to-healthy tissue uptake ratio associated with brief intraperitoneal ${ }^{125}$ I-35A7 radio-

Received Jun. 15, 2010; revision accepted Aug. 27, 2010.

For correspondence or reprints contact: Jean-Pierre Pouget, Institut de Recherche en Cancérologie de Montpellier, CRLC Val d'Aurelle, 34298 Montpellier Cedex 5, France.

E-mail: jean-pierre.pouget@valdorel.fnclcc.fr

COPYRIGHT @ 2010 by the Society of Nuclear Medicine, Inc. immunotherapy suggest that this method can be used in combination with radiation-synergistic drugs in the therapy of smallvolume peritoneal carcinomatosis after cytoreductive surgery.

Key Words: radioimmunotherapy; Auger electrons; peritoneal carcinomatosis

J Nucl Med 2010; 51:1748-1755

DOI: 10.2967/jnumed.110.080226

$\mathbf{P}$ eritoneal carcinomatosis is a common evolution of gastrointestinal or gynecologic tumors, or of primary peritoneal malignancies such as mesothelioma or peritoneal serous carcinoma. Peritoneal carcinomatosis has long been considered a terminal disease, having a median survival of 12-23 mo in patients with stage IV ovarian cancer, about 6 mo in colorectal carcinoma patients, 3 mo in gastric cancer patients, 2 mo in pancreatic cancer patients, and only 1.5 mo in patients with carcinomatosis from an unknown primary cancer $(1,2)$. The therapeutic approach is based on palliative systemic chemotherapy and surgery, according to symptoms, except in the case of ovarian cancer, for which complete cytoreduction is part of the standard therapeutic regimen. Twenty years ago, Sugarbaker introduced the use of cytoreductive surgery to resect the visible disease combined with hyperthermic intraperitoneal (IP) chemotherapy to treat the residual disease, as an innovative option for selected patients with peritoneal carcinomatosis $(3,4)$. The procedure for cytoreductive surgery depends on the extent of the peritoneal disease, and the chemotherapy protocols may include mitomycin $\mathrm{C}$, oxaliplatin, mitoxantrone, cisplatin, and irinotecan alone or in combination $(1,2)$. Hyperthermic IP chemotherapy can be performed using open- or closed-abdomen techniques, and perfusion may vary from 30 to $90 \mathrm{~min}$. Although the consensus about the ideal technique is not clear, cytoreductive surgery with hyperthermic IP chemotherapy has been shown to improve the survival of patients with peritoneal dissemi- 
nation from peritoneal pseudomyxoma, colorectal cancer, and diffuse peritoneal mesothelioma $(1,2,5,6)$. Mortality is relatively low, but morbidity does occur, from surgical complications or from toxicity (such as leukopenia; anemia; thrombopenia; and heart, liver, or kidney toxicity) due to the cytostatic agents $(1,2)$.

Several studies on rodents have shown that radioimmunotherapy (RIT) is an efficient adjuvant after cytoreductive surgery for peritoneal carcinomatosis (7-9). Several IP RIT studies using strong $\beta$ - or $\alpha$-emitters are ongoing in animals $(8-13)$. So far, 5 antibodies (against MUC1, CA-125, TAG72 , and gp38) have been conjugated to $4 \beta$-emitting radionuclides for IP RIT in patients with ovarian cancer (14). Based on previous encouraging results (15-19), a phase III randomized multicenter study was undertaken (20) in which the efficiency of conventional chemotherapy was compared with IP injection of ${ }^{90}$ Y-labeled HMGF1 murine $\mathrm{mAb}$ (anti-MUC1). However, no improvement in survival was observed after RIT, although peritoneal recurrence was significantly delayed. One explanation was that the irradiation dose delivered to the tumors was not high enough and that IP injection alone did not target all tumor deposits. Indeed, the induced toxicity by $\beta$ - and $\gamma$-emitters is a drawback for a treatment scheme using repeated injections. One alternative could be the use of short-range particles such as $\alpha$-emitters (21) or Auger electron emitters. We previously showed that anti-carcinoembryonic antigen (CEA) mAbs labeled with Auger electron emitters and intravenously injected into mice xenografted with cancer cells could significantly delay the growth of small peritoneal solid tumors $(7,23)$. We thus wanted to assess the efficiency and toxicity of ${ }^{125} \mathrm{I}-\mathrm{mAb}$ when used in IP RIT for mice bearing small peritoneal tumors. In addition, because the effects of IP RIT performed after hyperthermic IP chemotherapy have never, to our knowledge, been investigated, we decided to perform RIT using a closed peritoneal perfusion of ${ }^{125}$ I-labeled anti-CEA $\mathrm{mAb}$ for a short period $(1 \mathrm{~h})$, followed by extensive washing of the peritoneal cavity with $\mathrm{NaCl}$ (saline) solution to remove the unbound radioactivity. We call this procedure brief IP RIT.

\section{MATERIALS AND METHODS}

\section{Cell Line and $\mathrm{mAb}$}

The vulvar squamous carcinoma cell line A-431 expressing the epidermal growth factor receptor and transfected as previously described (22), with constructs encoding the CEA and luciferase genes, was used. The cells were grown as previously described (22). The noninternalizing murine IgG1k $35 \mathrm{~A} 7 \mathrm{mAb}$, specific for the CEA Gold 2 epitope, was used to target CEA. The irrelevant PX antibody was used for control experiments. PX is an IgG1 $\mathrm{mAb}$ that has been purified from the mouse myeloma MOPC 21 (24). PX and 35A7 were purified from mouse hybridoma ascitic fluids by ammonium sulfate precipitation followed by ion exchange chromatography on DE52 cellulose (Whatman).

\section{Radiolabeling}

${ }^{125}$ I was from Perkin Elmer, and mAbs were radiolabeled at the specific activity of $740 \mathrm{MBq} / \mathrm{mg}$ for RIT and biodistribution stud- ies, using the IODO-GEN (Pierce Chemical Co.) method and previously described methods (22). The immunoreactivity of ${ }^{125} \mathrm{I}-\mathrm{mAb}$ against CEA was assessed in vitro by direct binding assay (22). The binding percentage was determined by measuring the antigen-bound radioactivity after 2 washes with phosphatebuffered saline and was between $50 \%$ and $60 \%$.

\section{Animals}

Athymic nude mice (female, 6-8 wk old) were obtained from Charles River and were acclimated for $1 \mathrm{wk}$ before experimental use. They were housed at $22^{\circ} \mathrm{C}$ and $55 \%$ humidity with a lightdark cycle of $12 \mathrm{~h}$. Food and water were available ad libitum. The mice were force-fed with Lugol solution the day before RIT, and stable iodine was added to drinking water for the entire experimental period. Body weight was determined weekly, and the mice were clinically examined throughout the study. Hematologic toxicity was monitored for $70 \mathrm{~d}$ after the start of RIT, using the scil Vet abc system (SCIL Animal Care Co.). All animal experiments were performed in compliance with the guidelines of the French government and the standards of Institut National de la Santé et de la Recherche Médicale for experimental animal studies (agreement B34-172-27).

\section{RIT Experiments}

Mice were intraperitoneally grafted with $0.7 \times 10^{6}$ A-431 cells suspended in $0.3 \mathrm{~mL}$ of Dulbecco modified Eagle medium. Tumor growth was assessed $3 \mathrm{~d}$ after cell xenografting by bioluminescence imaging, which allowed the segregation of mice into homogeneous groups. Four days after grafting, the mice underwent IP or brief IP RIT.

The protocol for brief IP RIT was as follows. After being anesthetized by IP injection of a solution containing ketamine (100 mg/kg [Ketamine Panpharma; Panpharma]) and medetomidine ( $1 \mathrm{mg} / \mathrm{kg}$ [Dormitor; Pfizer]), the mice were intraperitoneally injected with either saline or ${ }^{125} \mathrm{I}-\mathrm{mAb}$ in a final volume of $5 \mathrm{~mL}$. One hour later, the peritoneal cavity was flushed with $25 \mathrm{~mL}$ of saline for $15 \mathrm{~min}$, using a perfusion system adapted from Aarts et al. (8). Typically, an inflow needle was placed in the upper abdominal cavity and 2 multiperforated catheters were inserted laterally through the abdominal wall to be used as outflows (Fig. 1A). Perfusion was done using a peristaltic pump (Harvard Apparatus). Once the peritoneal cavity had been washed, the catheters were removed and the mice weighed. They were then awakened by an IP injection of atipamezole $(2.5 \mathrm{mg} / \mathrm{kg}$ of body weight [Antisedan; Pfizer]).

The protocol for IP RIT consisted of a standard IP injection of either saline or ${ }^{125} \mathrm{I}-\mathrm{mAb}$ (final volume of $5 \mathrm{~mL}$ ) without washing of the peritoneal cavity, and intravenous (IV) RIT on day 7 or 11 was done conventionally, as previously described (22).

For brief IP RIT, one group of mice $(n=8)$ was treated with 5 $\mathrm{mL}$ of saline and another $(n=10)$ with $185 \mathrm{MBq}$ of ${ }^{125} \mathrm{I}-35 \mathrm{~A} 7$ $\mathrm{mAb}$. For IP RIT, the control group $(n=7)$ received a 5 -mL IP injection of saline, whereas the other group $(n=15)$ received one $37-\mathrm{MBq}$ IP injection of ${ }^{125} \mathrm{I}-35 \mathrm{~A} 7 \mathrm{mAb}$ on day 4 after grafting and another on day 7. For the combined brief IP and IV RIT, mice received brief IP ${ }^{125}$ I-35A7 RIT on day 4 after grafting and an IV 37-MBq injection of ${ }^{125} \mathrm{I}-35 \mathrm{~A} 7$ on day 7 or $11(n=9)$. To assess the nonspecific efficiency of ${ }^{125} \mathrm{I}-\mathrm{mAb}$, another group was treated with brief IP RIT using $185 \mathrm{MBq}$ of ${ }^{125} \mathrm{I}-\mathrm{PX}$ mAb followed by IV injection of $37 \mathrm{MBq}$ of ${ }^{125} \mathrm{I}-\mathrm{PX} \mathrm{mAb}$ on day 7. 


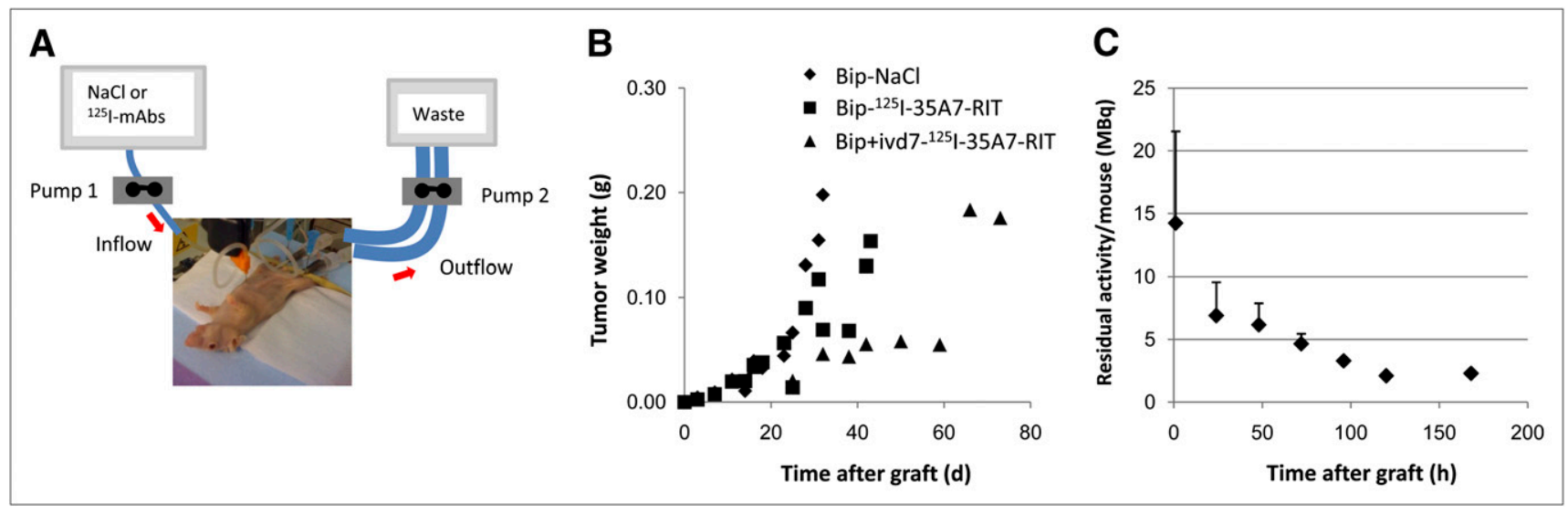

FIGURE 1. (A) Diagram of brief IP RIT perfusing system. (B) Graph of tumor growth. Mean tumor weight per mouse was determined from bioluminescence data obtained during RIT experiments and using calibration curve of Santoro et al. (22). (C) Graph of residual activity per mouse. Remaining activity in each mouse after washing of peritoneal cavity ( $1 \mathrm{~h}$ after injection) was determined by biodistribution study. Values are mean activity \pm SD. Bip $=$ brief IP; ivd7 = IV, day 7.

Tumor growth was followed weekly by bioluminescence imaging. Mice were sacrificed when the bioluminescence signal reached a value of $4.5 \times 10^{7}$ photons/s, corresponding to a total tumor weight of about $0.2-0.3 \mathrm{~g}$.

\section{Bioluminescence and SPECT/CT}

In vivo bioluminescence imaging was performed after IP injection of luciferin (0.1 mg of luciferin/g) as previously described (22). Whole-body SPECT/CT images were acquired at various times after brief IP ${ }^{125} \mathrm{I}-35 \mathrm{~A} 7 \mathrm{RIT}(0,1,24$, and $72 \mathrm{~h})$ with a 2-head multiplexing multipinhole NanoSPECT camera (Bioscan Inc.).

\section{Biodistribution Experiments to Mimic Brief IP RIT and IV RIT}

On day 1, 48 athymic nude mice were intraperitoneally grafted with $0.7 \times 10^{6} \mathrm{~A}-431$ cells that had been suspended in $0.3 \mathrm{~mL}$ of Dulbecco modified Eagle medium. Mice were divided into 2 groups to compare the biodistribution of ${ }^{125} \mathrm{I}-35 \mathrm{~A} 7 \mathrm{mAb}$ after brief IP RIT or IV RIT. To test IP biodistribution, one group of mice was treated with brief IP ${ }^{125}$ I-35A7 RIT according to the previously described protocol, but the injected solution contained $5.5 \mathrm{MBq}(740 \mathrm{MBq} / \mathrm{mg})$ of ${ }^{125} \mathrm{I}-35 \mathrm{~A} 7 \mathrm{mAb}$ completed with 243 $\mu \mathrm{g}$ of unlabeled $35 \mathrm{~A} 7 \mathrm{mAb}$ diluted in $5 \mathrm{~mL}$.

To test IV biodistribution, a second group was intravenously injected with a solution containing $185 \mathrm{kBq}(740 \mathrm{MBq} / \mathrm{mg})$ of ${ }^{125} \mathrm{I}-35 \mathrm{~A} 7 \mathrm{mAb}$ completed with $50 \mu \mathrm{g}$ of unlabeled $35 \mathrm{~A} 7 \mathrm{mAb}$ diluted in $300 \mu \mathrm{L}$ of saline solution.

The mice were sacrificed at 1, 24, 48, 72, 96, 144, and $168 \mathrm{~h}$ after treatment. At each time point, animals were anesthetized; bioluminescence images acquired; and the animals euthanized, bled, and dissected. Blood and healthy organs were weighed. The size of tumor nodules was determined, the tumor volume calculated, and thereby the tumor weight calculated on the basis of a density of $1.05 \mathrm{~g} / \mathrm{cm}^{3}$ as described by Santoro et al. (22). The radioactivity uptake during the biodistribution experiments was then measured for tumor nodules and for all organs, using a $\gamma$-well counter. The percentage of injected activity per gram of tissue, corrected for radioactive decay, was calculated for IV ${ }^{125} \mathrm{I}-35 \mathrm{~A} 7$ biodistribution. For brief IP ${ }^{125} \mathrm{I}-35 \mathrm{~A} 7$ biodistribution, results were expressed as percentage of the remaining activity per gram of tissue immediately after the peritoneal wash (i.e., $1 \mathrm{~h}$ after injection).

\section{Tumor Weight Assessment During RIT Experiments}

Accurate, direct weighing of peritoneal tumors was not possible in RIT experiments because of the high activities and because the mice would have to be sacrificed; therefore, weights were calculated from the weekly bioluminescence imaging data. For this purpose, biodistribution experiments were used to determine the calibration curve between the bioluminescence signal of tumors and their size. Typically, before sacrifice, tumors were imaged by bioluminescence and the corresponding signal (photon/s) was correlated with the calculated tumor weight $(\mathrm{g})$ determined by direct measurement of the size of tumor nodules, as described previously (22). The calibration curve was similar to that determined in our previous work (22).

\section{Uptake of Radioactivity per Organ and Tumor and Dosimetry}

The uptake of radioactivity per tissue $(\mathrm{Bq})$ in RIT experiments was extrapolated from the uptake per tissue measured during the biodistribution experiments. Because the activities used in RIT experiments were 33 times higher than those used in the biodistribution analysis (185 MBq vs. $5.5 \mathrm{MBq}$ ) for the same amount of injected $\mathrm{mAb}(250 \mu \mathrm{g})$, all uptake values from the biodistribution experiments were multiplied by 33 to mimic therapeutic conditions. We considered that the weight of healthy tissues did not change during the study and that they did not differ between RIT and biodistribution experimental conditions. We confirmed that this assumption was true also for tumor nodules during the first week after injection. Therefore, the 33-fold correction was enough to distinguish uptake of radioactivity during the RIT experiments from uptake of radioactivity during the biodistribution experiments. The total cumulative decays per tissue, $\tilde{A}_{r s}$, were calculated by measuring the area under the curves for the uptake of radioactivity during the RIT experiments. Following the MIRD formalism (25), the resulting values were multiplied by the $S$ factor for determining the irradiation doses as determined in our previous work (22).

\section{Statistical Analysis}

Kaplan-Meier survival estimates were calculated from the date of the xenograft until the date of the event of interest (i.e., a bioluminescence value of $4.5 \times 10^{7}$ photons/s) and compared with the 
Log-rank test. Statistical analysis was performed using STATA 10.0 software.

\section{RESULTS}

\section{Tumor Growth}

On day 4 after grafting (just before treatment began), 5-6 nodules per mouse with a mean diameter of about 1.5-2 $\mathrm{mm}$ were detected by bioluminescence imaging. This size corresponded to a mean tumor weight of $1.2 \pm 0.9 \times 10^{-2} \mathrm{~g}$. In the brief IP saline group, tumors grew exponentially and all mice were sacrificed before day 40 after grafting (Fig. 1B). A similar growth rate was observed in the group treated by brief IP + IV day $7{ }^{125}$ I-PX RIT (data not shown). The longest delay in tumor growth was observed in the group treated by brief IP + IV day $7^{125}$ I-35A7 RIT. Intermediary tumor growth kinetics were reported for the groups treated by brief IP ${ }^{125}$ I-35A7 RIT (Fig.1B), brief IP + IV day 11 ${ }^{125} \mathrm{I}-35 \mathrm{~A} 7 \mathrm{RIT}$, and IP + IP day $7{ }^{125} \mathrm{I}-35 \mathrm{~A} 7$ RIT (data not shown).

Washing of the peritoneal cavity with saline slowed tumor growth in the brief IP saline group, compared with the IP saline group (data not shown). This observation was further strengthened by the analysis of survival data; survival was significantly lower after IP saline $(P<$ 0.001, compare Fig. 2A with Fig. 2B).

\section{Toxicity of Brief IP RIT Using ${ }^{125} \mathrm{I}-\mathrm{mAb}$}

The residual activity per mouse was about $14.2 \pm 7.3$ $\mathrm{MBq}$ immediately after the wash with saline solution and

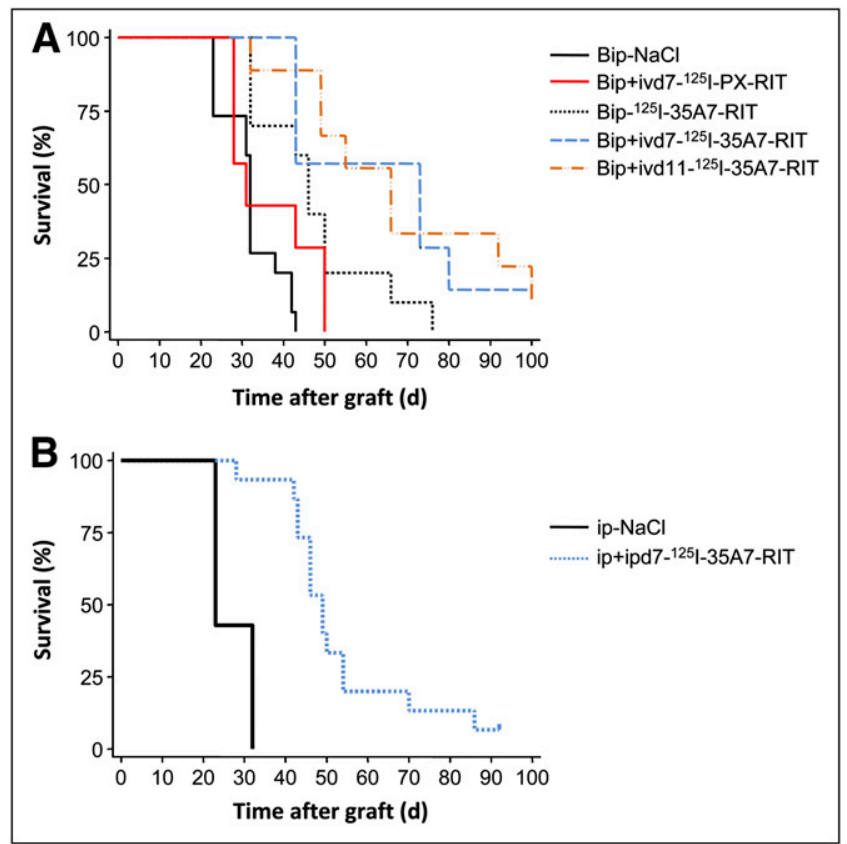

FIGURE 2. Graphs of survival curves for athymic mice bearing IP A-431 tumor cell xenografts and treated with ${ }^{125} \mathrm{I}-\mathrm{mAb}$. Mice were sacrificed when bioluminescence signal reached $4.5 \times 10^{7}$ photons/s. Censored mice are indicated by vertical bars. Survival rates were estimated using Kaplan-Meier method. Bip = brief IP; ipd7 = IP, day $7 ; \operatorname{ivd} 7=\mathrm{IV}$, day $7 ; \operatorname{ivd} 11=\mathrm{IV}$, day 11 . dropped to $2.1 \pm 0.7 \mathrm{MBq}$ at $120 \mathrm{~h}$ after brief IP RIT (Fig. 1C). These results indicate that about $7.6 \%$ of the injected activity was effectively kept within the peritoneal cavity. No weight loss was observed after brief IP RIT (Supplemental Fig. 1). These results suggest that brief IP RIT is well tolerated by mice. However mild, transient hematologic toxicity was reported in all treated mice, in comparison to the control brief IP saline group (Fig. 3 and Supplemental Fig. 2). In the brief IP ${ }^{125}$ I-35A7 RIT group (Fig. 3), the nadir for white blood cells was reached between days 7 and 10 (around -20\%) after grafting and was due mainly to a decrease in lymphocyte and monocyte levels (-40\%) (Supplemental Fig. 2). The lowest platelet point occurred slightly later (days 10-15, -30\%), whereas no modification in red blood cells was observed. Most values returned to normal around day 39. In mice that received an IV injection of ${ }^{125} \mathrm{I}-\mathrm{mAb}$ on day 7 (brief IP + IV day 7 ${ }^{125}$ I-35A7 RIT and brief IP + IV day $7{ }^{125}$ I-PX RIT), the decrease in white blood cells was more pronounced and prolonged (about $-70 \%$ on day 22 ; Fig. 3 ). When the IV injection was done on day 11 (brief IP + IV day $11^{125} \mathrm{I}$ 35A7 RIT) after grafting, the hematologic toxicity was in the same range but more prolonged, and values had not yet returned to baseline by day 39 (Fig. 3 and Supplemental Fig. 2). The ratio for day 52 could not be calculated, since most of the mice in the brief IP saline group had to be sacrificed because of tumor growth.

The similar effects observed for mice receiving brief IP + IV day $7{ }^{125}$ I-35A7 RIT and brief IP + IV day $7{ }^{125} \mathrm{I}-\mathrm{PX}$ RIT suggest that the hematologic toxicity was mainly due to nonspecific irradiation, including soft X-rays or the most energetic electrons emitted by ${ }^{125} \mathrm{I}$. In addition, IP + IP day 7 125I-35A7 RIT (Fig. 3 and Supplemental Fig. 2) caused lower hematologic toxicity than did brief IP + IV day 7 ${ }^{125}$ I-35A7 RIT, despite the higher activities detected in IP + IP day $7^{125}$ I-35A7 RIT mice. Indeed, during the latter IP RIT, $74 \mathrm{MBq}(2 \times 37 \mathrm{MBq})$ were effectively injected and available for diffusion toward the blood compartment. By

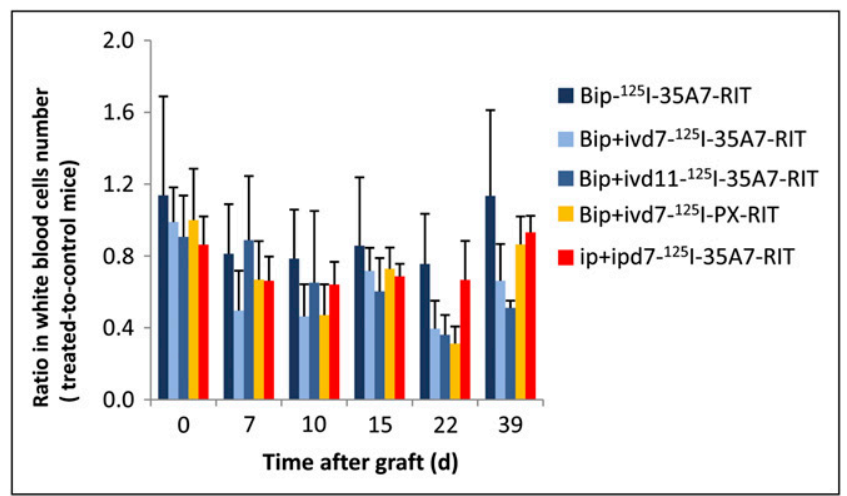

FIGURE 3. Bar graft of hematologic toxicity. White blood cell number was monitored at various times (0-39 d) after treatment and expressed as ratio of treated mice to control mice. Bip $=$ brief $\mathrm{IP} ; \mathrm{ipd} 7=\mathrm{IP}$, day 7 ; ivd7 = IV, day 7; ivd11 = IV, day 11 . 
contrast, for brief IP + IV RIT, of the $185 \mathrm{MBq}$ used, only 14.2 $\mathrm{MBq}$ were remaining in the mouse immediately after washing of the peritoneal cavity. This activity was completed by IV injection of $37 \mathrm{MBq}$; that is, a final total activity of $51.2 \mathrm{MBq}$ was effectively remaining in the mouse. This result suggests that the high activity of 185 $\mathrm{MBq}$ maintained for $1 \mathrm{~h}$ during brief IP RIT is mainly responsible for the hematologic toxicity.

\section{Improved Survival with Brief IP 125I-35A7 RIT}

Mice were sacrificed when the bioluminescence signal reached $4.5 \times 10^{7}$ photons/s, corresponding to a mean tumor weight of about $0.2-0.3 \mathrm{~g}$. The median survival was about $32 \mathrm{~d}$ in the brief IP saline group but significantly increased $(P<0.001)$ to $46 \mathrm{~d}$ in the group treated with brief IP ${ }^{125} \mathrm{I}-35 \mathrm{~A} 7 \mathrm{RIT}$ (Fig. 2A). This value was significantly improved $(P<0.001)$ when brief IP RIT was combined with an IV injection on day 11 (brief IP + IV day $11^{125} \mathrm{I}-$ 35A7 RIT; median survival $=66 \mathrm{~d}$ ) or day 7 (brief IP + IV day $7^{125}$ I-35A7 RIT; median survival = $73 \mathrm{~d}$ ) (Fig. 2A). Moreover, 1 mouse in each of these 2 groups was cured by the end of the observation period of $100 \mathrm{~d}$. Mice treated by brief IP + IV day $7{ }^{125}$ I-PX RIT had a median survival of $31 \mathrm{~d}$-somewhat similar to the control brief IP saline group $(P=0.188)$ (Fig. 2A), suggesting the absence of toxicity and the efficiency of ${ }^{125} \mathrm{I}$ when unbound to cells. Finally, median survival was $49 \mathrm{~d}$ in the IP + IP day $7^{125} \mathrm{I}-35 \mathrm{~A} 7$ RIT mice (Fig. 2B) and only $23 \mathrm{~d}$ in the IP + IP day 7 saline RIT control group $(P<0.001)$. Moreover one of the IP + IP day $7{ }^{125}$ I-35A7 RIT mice was cured by the end of the period of interest.

\section{SPECT/CT After Brief IP 125I-35A7 RIT}

Whole-body SPECT/CT showed that after injection, radioactivity was homogeneously distributed in the peritoneal cavity (Fig. 4A). After washing of the peritoneal cavity, the radioactivity concentrated at tumor nodules up to $3 \mathrm{~d}$ after injection (Fig. 4A), as indicated by the good fitting with the bioluminescence signals (Fig. 4B).

\section{Improved Tumor-to-Healthy Tissues Uptake Ratio with Brief IP 125I-35A7 RIT}

The biodistribution study confirmed the strong uptake of ${ }^{125} \mathrm{I}-35 \mathrm{~A} 7 \mathrm{mAb}$ by tumor nodules (Fig. 5A). The percentage of remaining activity per gram of tumor immediately after washing of the peritoneal cavity ranged from $72.1 \% \pm$ $30.2 \%$ at $1 \mathrm{~h}$ to $20.5 \% \pm 4.8 \%$ at $168 \mathrm{~h}$. These values were much higher than the peak value of $27.8 \% \pm 7.2 \%$ of injected activity per gram of tumor determined at $96 \mathrm{~h}$ after a single IV injection of $37 \mathrm{MBq}(740 \mathrm{MBq} / \mathrm{mg})$, typical of IV ${ }^{125}$ I-35A7 RIT (Fig. 5B). Moreover, the uptake of radioactivity by healthy organs was higher after IV injection than after brief IP ${ }^{125} \mathrm{I}-35 \mathrm{~A} 7$ RIT. For instance, in brief IP ${ }^{125} \mathrm{I}-35 \mathrm{~A} 7$ biodistribution mice, the peak value in blood at $48 \mathrm{~h}$ was $12.2 \% \pm 3.2 \%$ of remaining activity per gram, whereas this peak value was about $28.1 \% \pm 2.4 \%$ of injected activity per gram in the IV ${ }^{125} \mathrm{I}-35 \mathrm{~A} 7$ biodistribution group at $1 \mathrm{~h}$ after injection (Fig. 5B). These results suggest that brief IP ${ }^{125} \mathrm{I}-35 \mathrm{~A} 7$ RIT improves tumor targeting and that healthy tissues are more protected, compared with IV injection.

\section{Cumulated Uptake of Radioactivity After Brief IP 125I-35A7 RIT and Dosimetry}

From the biodistribution data, we expressed the uptake of radioactivity of ${ }^{125} \mathrm{I}-35 \mathrm{~A} 7 \mathrm{mAb}$ by healthy organs and tumor nodules as a function of time (Figs. 6A and 6B and Supplemental Fig. 3).

The cumulated uptake of radioactivity $\left(\tilde{A}_{r s}\right)$ was then determined by calculating the area under the curves shown in Figures 6A and 6B together with Supplemental Figure 3. According to the MIRD formalism, the mean absorbed irradiation dose per organ was calculated by multiplying $\tilde{A}_{r s}$ by the $\mathrm{S}$ value corresponding to ${ }^{125} \mathrm{I}$. The mean absorbed irradiation dose by tumors was $11.6 \mathrm{~Gy}$ after brief IP ${ }^{125} \mathrm{I}-35 \mathrm{~A} 7 \mathrm{RIT}$ and $16.7 \mathrm{~Gy}$ after IV ${ }^{125} \mathrm{I}-35 \mathrm{~A} 7 \mathrm{RIT}$. The irradiation dose delivered to healthy organs after brief IP ${ }^{125}$ I-35A7 RIT was much lower than that after IV ${ }^{125}$ I-35A7 RIT: for instance, blood received about 1.9 Gy after brief IP ${ }^{125} \mathrm{I}-35 \mathrm{~A} 7 \mathrm{RIT}$ and about 9.8 Gy after IV ${ }^{125} \mathrm{I}-35 \mathrm{~A} 7 \mathrm{RIT}$ (Fig. 6C). The irradiation dose to the other organs did not exceed 1 Gy after brief IP ${ }^{125}$ I-35A7 RIT and 4.2 Gy after IV ${ }^{125}$ I-35A7 RIT.

\section{DISCUSSION}

In the present study, we investigated the feasibility, toxicity, and efficacy of brief IP RIT using a ${ }^{125} \mathrm{I}-\mathrm{mAb}$ directed against CEA. This protocol is based on the hyperthermic IP chemotherapy methodology, although hyperther-

FIGURE 4. Mouse treated by brief IP 125I35A7 RIT (185 MBq) on day 4 after grafting. (A) SPECT/CT images obtained immediately after injection $(0 \mathrm{~h})$ and at 1,24 , and $72 \mathrm{~h}$ after washing of peritoneal cavity with saline solution. (B) Bioluminescence image obtained $4 \mathrm{~d}$ after grafting, just before RIT.
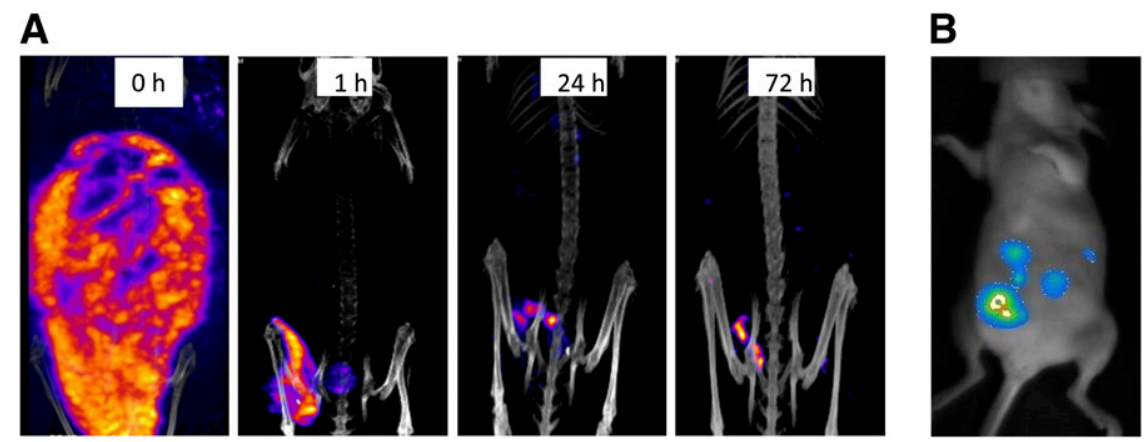


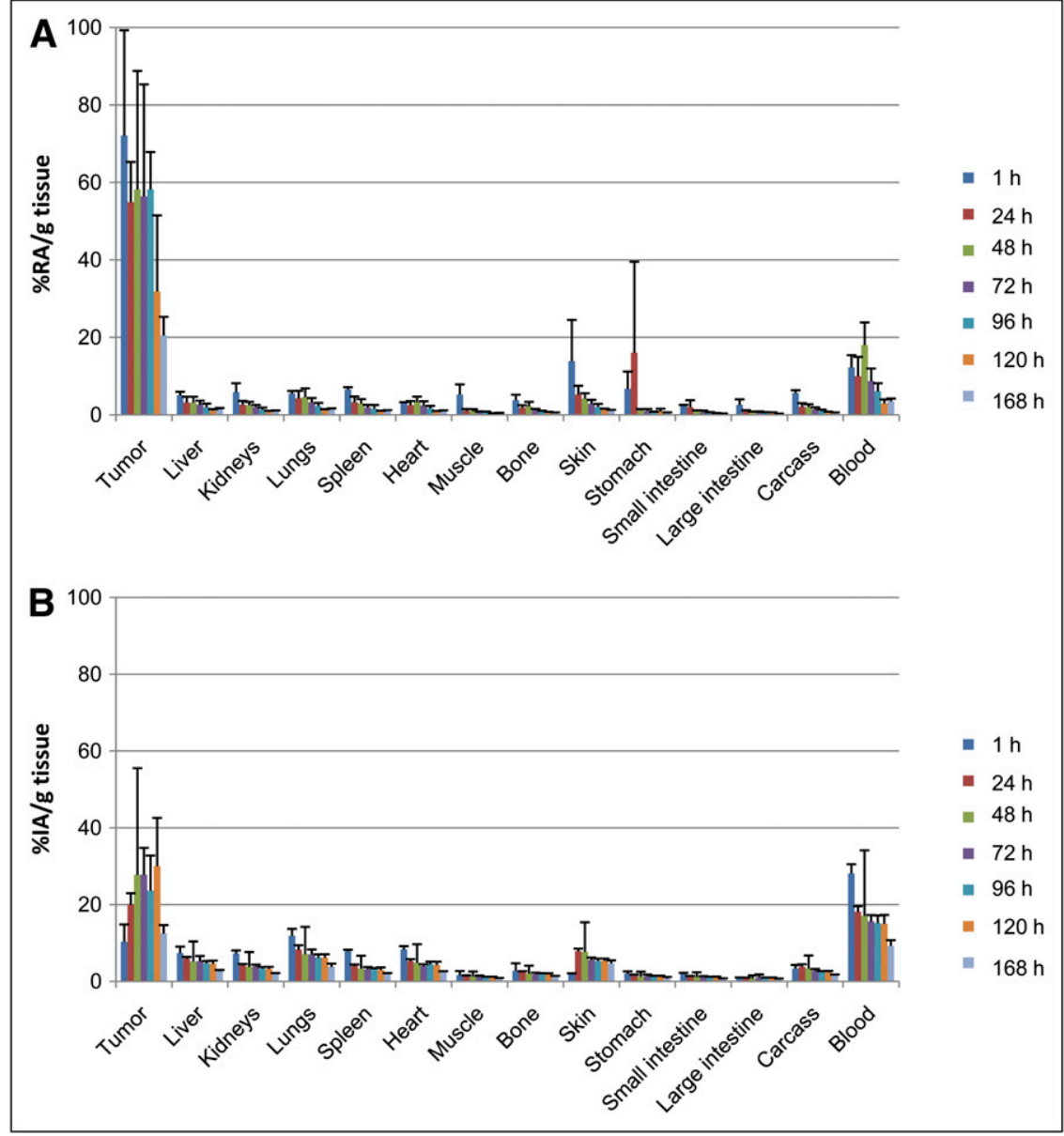

FIGURE 5. Graphs of biodistribution analysis in athymic nude mice bearing IP A-431 tumor cell xenografts. (A) In mice treated by brief IP ${ }^{125}$ I-35A7 RIT, percentage of remaining activity per gram of tissue in healthy organs and tumors immediately after washing of peritoneal cavity. (B) In mice treated by IV ${ }^{125}$ I-35A7 RIT, percentage of injected activity per gram of tissue in healthy organs and tumors. Four mice were analyzed at each time point. \%IA = percentage injected activity; \%RA = percentage remaining activity. mia was not associated with brief IP RIT. Our results indicate that about $7 \%$ of the injected activity, corresponding to about 14.2 MBq, was retained in the peritoneal cavity after washing. Mild and transient blood toxicity was observed and was mainly due to nonspecific irradiation (possibly soft $\mathrm{X}$-rays and more energetic electrons emitted by ${ }^{125} \mathrm{I}$ ) that occurred during the 1 -h peritoneal incubation with high activities of ${ }^{125} \mathrm{I}-\mathrm{mAb}$. However, we estimated that in patients, about $300 \mu \mathrm{Gy}$ would be delivered by such an external irradiation at the sacral level (data not shown). Therefore, the hematologic toxicity is expected to be much lower than in mice.

SPECT/CT of mice at different time points after brief IP ${ }^{125} \mathrm{I}-35 \mathrm{~A} 7 \mathrm{RIT}$, and the biodistribution experiments, indicate that radioactivity was concentrated in tumor nodules, whereas the irradiation dose to healthy organs by brief IP ${ }^{125} \mathrm{I}-35 \mathrm{~A} 7$ RIT was generally much lower than with IV ${ }^{125} \mathrm{I}-$ 35A7 RIT and did not exceed 1 Gy. In addition, although the irradiation dose delivered to the tumors was lower after brief IP ${ }^{125}$ I-35A7 RIT than after IV ${ }^{125}$ I-35A7 RIT, the tumor-to-blood irradiation dose ratio was about 5 for brief IP ${ }^{125}$ I-35A7 RIT and 1.7 for IV ${ }^{125}$ I-35A7 RIT. These results indicate that brief IP ${ }^{125} \mathrm{I}-35 \mathrm{~A} 7$ RIT protects healthy tissues while delivering significant irradiation doses to tumors, confirming the generally described advantage of
IP over IV RIT for peritoneal disease in terms of concentration and tolerance (14). Therefore, although reducing the potential blood reservoir of ${ }^{125} \mathrm{I}-\mathrm{mAb}$ in comparison to IV RIT, the brief IP RIT procedure, which includes washing of the peritoneal cavity, eliminates undesirable radioactivity and therefore is less toxic.

The median survival of xenografted mice was significantly improved after brief IP ${ }^{125}$ I-35A7 RIT alone (46 d) in comparison to controls ( $32 \mathrm{~d}$ ). However, the combination of brief IP ${ }^{125}$ I-35A7 RIT on day 4 and IV ${ }^{125}$ I-35A7 RIT on day 7 further increased survival to $73 \mathrm{~d}$. In our previous study (22), median survival was $59 \mathrm{~d}$ (vs. $19 \mathrm{~d}$ for controls) for mice treated with 2 IV injections of $37 \mathrm{MBq}$ of ${ }^{125} \mathrm{I}-$ $35 \mathrm{~A} 7 \mathrm{mAb}$. Assuming that the transient hematologic toxicity we observed after brief IP ${ }^{125} \mathrm{I}-35 \mathrm{~A} 7 \mathrm{RIT}$ is effectively due to the initial $1-\mathrm{h}$ incubation with ${ }^{125} \mathrm{I}-\mathrm{mAb}$ in the peritoneal cavity, repeated IV injections of ${ }^{125} \mathrm{I}-\mathrm{mAb}$ could be planned after brief IP RIT-or the combination of brief IP ${ }^{125}$ I-35A7 RIT and chemotherapy might be envisagedwithout a significant increase of toxicity, as we observed in the brief IP + IV day $7^{125}$ I-35A7 RIT group. The median survival increased to $49 \mathrm{~d}$ also after 2 IP injections of anti-CEA mAb (IP + IP day $7{ }^{125}$ I-35A7 RIT), with low associated hematologic toxicity. However, the effect on survival was weaker than the improvement obtained after brief 
FIGURE 6. (A and B) Graphs showing uptake of radioactivity in tissue after brief IP 125I-35A7 RIT (A) or IV ${ }^{125}$ I-35A7 RIT (B), determined using values obtained during biodistribution experiments (Fig. 5). For clarity, only most representative results are shown, and uptake values that are out of scale $(0-1.5 \times$ $10^{6} \mathrm{~Bq}$ ) are shown in Supplemental Figure 3. (C) Graph of mean absorbed irradiation dose for brief IP 125I-35A7 RIT and IV 125I-35A7 RIT. From uptake of radioactivity curves, total cumulative decay per tissue, $\tilde{A}_{r s}$, was calculated from area under each curve. $\tilde{A}_{r s}$, was then multiplied by $19.483 \mathrm{ke} \mathrm{V}$, which corresponds to mean energy delivered at each ${ }^{125}$ decay. Bip $=$ brief IP.

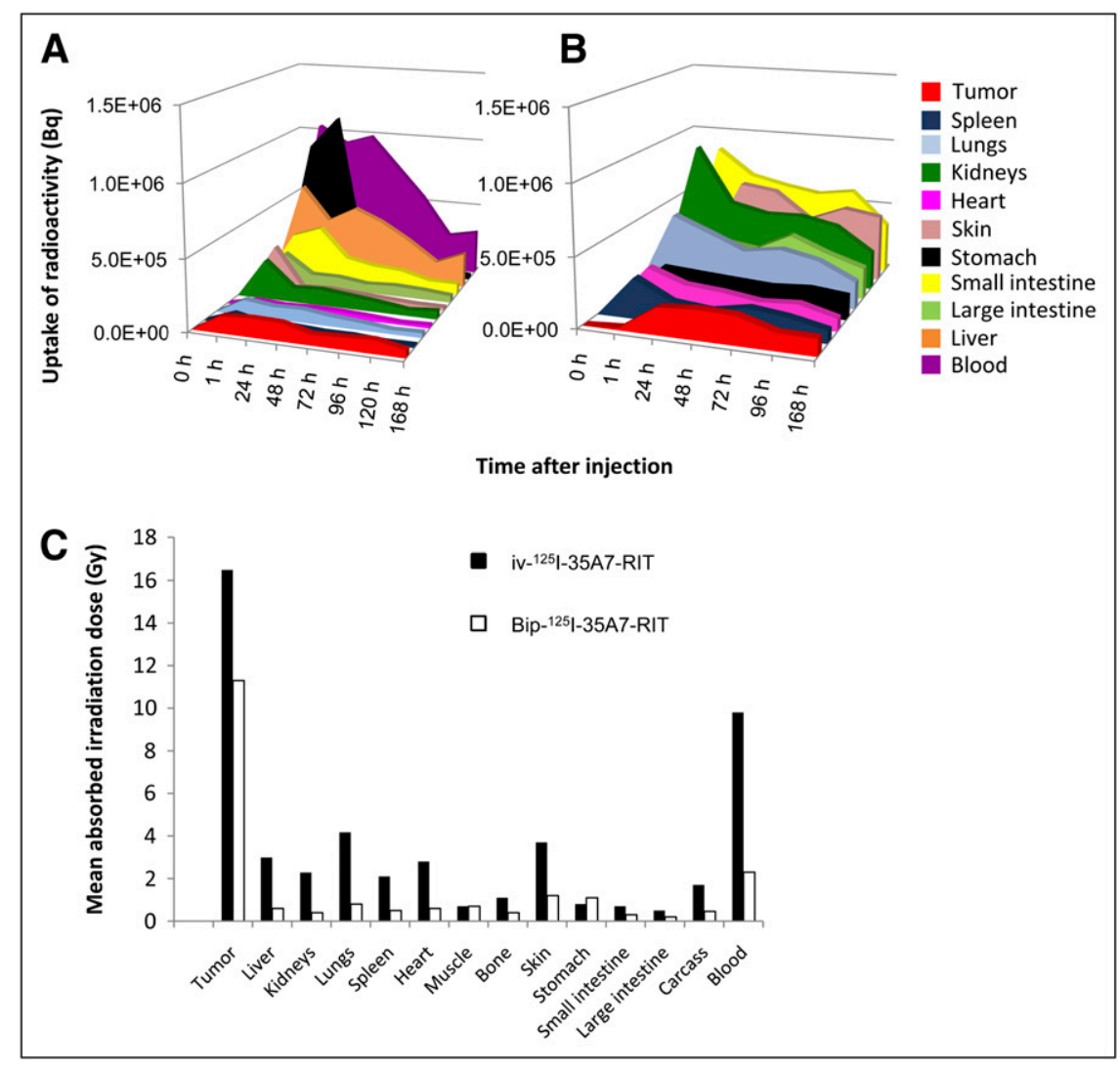

IP + IV day $7{ }^{125}$ I-35A7 RIT (73 vs. $49 \mathrm{~d}$; whereas controls had a median survival of 32 and $23 \mathrm{~d}$, respectively). Moreover, the uptake of radioactivity by healthy tissues during brief IP RIT was low and was probably much lower than during IP RIT. In addition, the combination of brief IP RIT and IV RIT takes advantage of the better peritoneal tumor uptake obtained through the IP route, and the delayed IV injection may allow reaching pockets of cancer cells that were not targeted by brief IP RIT (26).

Completeness of resection and tumor load are the most important predictors of long-term survival after cytoreductive surgery and hyperthermic IP chemotherapy. In the present study, we targeted tumor nodules of about $1.2 \pm 0.9$ $\times 10^{-2} \mathrm{~g}$. It was shown with $\beta$-emitters that RIT must be dedicated to small solid tumors (27). The size parameter is even more crucial when low-energy electron emitters are used, because the path length of electrons emitted by ${ }^{125} \mathrm{I}$ ranges from nanometers to about $20 \mu \mathrm{m}$, whereas in peritoneal carcinomatosis, tumor nodules have a diameter of several millimeters. Our results can be compared with the work of Aarts et al., who found that when RIT was combined with cytoreductive surgery, median survival increased from 57 to $97 \mathrm{~d}$ in rats that had CC-531 colon carcinoma tumor xenografts of a few millimeters and were treated with a single $74-\mathrm{MBq}$ IP injection of ${ }^{177} \mathrm{Lu}-$ labeled MG1 mAb $(8,9)$. The low toxicity for healthy tissue of Auger electron emitters such as ${ }^{125} \mathrm{I}$ is expected to be an advantage for the treatment of small peritoneal carcinomatosis, especially because of the potential for combining RIT with radiation- synergistic chemotherapy, such as taxol $(28,29)$, or drugs targeting the tumor microenvironment (30-32). In addition, repeated ${ }^{125} \mathrm{I}-\mathrm{mAb}$ injections will also be made possible. The precise mechanisms by which ${ }^{125}$ I-anti CEA mAb efficiently delays tumor growth remain to be elucidated. Considering that most of the electron dose generated by ${ }^{125} \mathrm{I}$ decay is extremely localized, most of the irradiation dose must be delivered to the cell membrane when targeted by ${ }^{125} \mathrm{I}$-antiCEA mAb. Therefore, we can hypothesize that the cell membrane is an important target of high-LET Auger electrons.

\section{CONCLUSION}

Our data confirm the efficiency of ${ }^{125} \mathrm{I}$-anti CEA mAb in killing tumor cells. Brief IP RIT is accompanied by a strong tumor-to-healthy tissue ratio that allows an increase in injected activities while protecting healthy tissues. This is reinforced by the use of Auger electron emitters such as ${ }^{125} \mathrm{I}$, as they decrease nonspecific irradiation. All these features might allow the use of repeated injections of radiosynergistic agents. Therefore, our results suggest that brief IP RIT with ${ }^{125}$ I-labeled anti-CEA mAb might be a complementary tool in the therapy of small solid tumors after cytoreductive surgery.

\section{ACKNOWLEDGMENT}

This work was supported by the Institut National du Cancer, grant R09080FF/RPT09005FFA. We thank Imade Ait Arsa for animal care and involvement in the experiments. labeled antibodies or the combination of RIT with radiation- 


\section{REFERENCES}

1. Glehen O, Mohamed F, Gilly FN. Peritoneal carcinomatosis from digestive tract cancer: new management by cytoreductive surgery and intraperitoneal chemohyperthermia. Lancet Oncol. 2004;5:219-228.

2. Glockzin G, Schlitt HJ, Piso P. Peritoneal carcinomatosis: patients selection, perioperative complications and quality of life related to cytoreductive surgery and hyperthermic intraperitoneal chemotherapy. World J Surg Oncol. 2009;7:5.

3. Sugarbaker PH. Comprehensive management of peritoneal surface malignancy using cytoreductive surgery and perioperative intraperitoneal chemotherapy: the Washington Cancer Institute approach. Expert Opin Pharmacother. 2009; 10:1965-1977.

4. Sugarbaker PH, Cunliffe WJ, Belliveau J, et al. Rationale for integrating early postoperative intraperitoneal chemotherapy into the surgical treatment of gastrointestinal cancer. Semin Oncol. 1989;16:83-97.

5. Elias D, Gilly F, Boutitie F, et al. Peritoneal colorectal carcinomatosis treated with surgery and perioperative intraperitoneal chemotherapy: retrospective analysis of 523 patients from a multicentric French study. J Clin Oncol. 2010;28:63-68.

6. Elias D, Gilly F, Quenet F, et al. Pseudomyxoma peritonei: a French multicentric study of 301 patients treated with cytoreductive surgery and intraperitoneal chemotherapy. Eur J Surg Oncol. 2010;36:456-462.

7. Koppe MJ, Bleichrodt RP, Oyen WJG, Boerman OC. Radioimmunotherapy and colorectal cancer. Br J Surg. 2005;92:264-276.

8. Aarts F, Hendriks T, Boerman OC, Koppe MJ, Oyen WJG, Bleichrodt RP. A comparison between radioimmunotherapy and hyperthermic intraperitoneal chemotherapy for the treatment of peritoneal carcinomatosis of colonic origin in rats. Ann Surg Oncol. 2007;14:3274-3282.

9. Aarts F, Bleichrodt R, de Man B, Lomme R, Boerman O, Hendriks T. The effects of adjuvant experimental radioimmunotherapy and hyperthermic intraperitoneal chemotherapy on intestinal and abdominal healing after cytoreductive surgery for peritoneal carcinomatosis in the rat. Ann Surg Oncol. 2008;15:3299-3307.

10. Koppe MJ, Soede AC, Pels W, et al. Experimental radioimmunotherapy of small peritoneal metastases of colorectal origin. Int J Cancer. 2003;106:965-972.

11. Kinuya S, Yokoyama K, Fukuoka M, et al. Intraperitoneal radioimmunotherapy to treat the early phase of peritoneal dissemination of human colon cancer cells in a murine model. Nucl Med Commun. 2007;28:129-133.

12. Elgqvist J, Andersson H, Bäck T, et al. Therapeutic efficacy and tumor dose estimations in radioimmunotherapy of intraperitoneally growing OVCAR-3 cells in nude mice with ${ }^{211}$ At-labeled monoclonal antibody MX35. J Nucl Med. 2005; 46:1907-1915.

13. Milenic DE, Brady ED, Garmestani K, Albert PS, Abdulla A, Brechbiel MW. Improved efficacy of alpha-particle-targeted radiation therapy: dual targeting of human epidermal growth factor receptor- 2 and tumor-associated glycoprotein 72 . Cancer. 2010;116:1059-1066.

14. Meredith RF, Buchsbaum DJ, Alvarez RD, LoBuglio AF. Brief overview of preclinical and clinical studies in the development of intraperitoneal radioimmunotherapy for ovarian cancer. Clin Cancer Res. 2007;13:5643s-5645s.

15. Alvarez RD, Huh WK, Khazaeli MB, et al. Phase I study of combined modality ${ }^{90}$ yttrium-CC49 intraperitoneal radioimmunotherapy for ovarian cancer. Clin Cancer Res. 2002;8:2806-2811.
16. Epenetos AA, Hird V, Lambert H, Mason P, Coulter C. Long term survival of patients with advanced ovarian cancer treated with intraperitoneal radioimmunotherapy. Int J Gynecol Cancer. 2000;10:44-46.

17. Meredith RF, Partridge EE, Alvarez RD, et al. Intraperitoneal radioimmunotherapy of ovarian cancer with lutetium-177-CC49. J Nucl Med. 1996;37: 1491-1496.

18. Hird V, Maraveyas A, Snook D, et al. Adjuvant therapy of ovarian cancer with radioactive monoclonal antibody. Br J Cancer. 1993;68:403-406.

19. Nicholson S, Gooden CS, Hird V, et al. Radioimmunotherapy after chemotherapy compared to chemotherapy alone in the treatment of advanced ovarian cancer: a matched analysis. Oncol Rep. 1998;5:223-226.

20. Verheijen RH, Massuger LF, Benigno BB, et al. Phase III trial of intraperitoneal therapy with yttrium-90-labeled HMFG1 murine monoclonal antibody in patients with epithelial ovarian cancer after a surgically defined complete remission. J Clin Oncol. 2006;24:571-578.

21. Andersson H, Cederkrantz E, Bäck T, et al. Intraperitoneal alpha-particle radioimmunotherapy of ovarian cancer patients: pharmacokinetics and dosimetry of ${ }^{211}$ At-MX35 F(ab')2—a phase I study. J Nucl Med. 2009;50:1153-1160.

22. Santoro L, Boutaleb S, Garambois V, et al. Noninternalizing monoclonal antibodies are suitable candidates for ${ }^{125} \mathrm{I}$ radioimmunotherapy of small-volume peritoneal carcinomatosis. J Nucl Med. 2009;50:2033-2041.

23. Pouget JP, Santoro L, Raymond L, et al. Cell membrane is a more sensitive target than cytoplasm to dense ionization produced by Auger electrons. Radiat Res. 2008;170:192-200.

24. Köhler G, Howe S, Milstein C. Fusion between immunoglobulin-secreting and nonsecreting myeloma cell lines. Eur J Immunol. 1976;6:292-295.

25. Loevinger R, Budinger T, Watson E. MIRD Primer for Absorbed Dose Calculations. Revised ed. New York, NY: Society of Nuclear Medicine; 1991.

26. Goldenberg DM. Adjuvant and combined radioimmunotherapy: problems and prospects on the road to Minerva. J Nucl Med. 2006;47:1746-1748.

27. Ychou M, Azria D, Faurous P, et al. Adjuvant radioimmunotherapy trial with iodine-131-labeled anti-CEA monoclonal antibody $\mathrm{F} 6 \mathrm{~F}\left(\mathrm{ab}^{\prime}\right) 2$ after resection of liver metastases from colorectal cancer. Clin Cancer Res. 2008;14:3487-3493.

28. DeNardo SJ, Kukis DL, Kroger LA, et al. Synergy of Taxol and radioimmunotherapy with yttrium-90-labeled chimeric L6 antibody: efficacy and toxicity in breast cancer xenografts. Proc Natl Acad Sci USA. 1997;94:4000-4004.

29. Supiot S, Gouard S, Charrier J, et al. Mechanisms of cell sensitization to alpha radioimmunotherapy by doxorubicin or paclitaxel in multiple myeloma cell lines. Clin Cancer Res. 2005;11:7047s-7052s.

30. Burke PA, DeNardo SJ, Miers LA, Lamborn KR, Matzku S, DeNardo GL. Cilengitide targeting of alpha(v)beta(3) integrin receptor synergizes with radioimmunotherapy to increase efficacy and apoptosis in breast cancer xenografts. Cancer Res. 2002;62:4263-4272.

31. Pedley RB, El-Emir E, Flynn AA, et al. Synergy between vascular targeting agents and antibody-directed therapy. Int J Radiat Oncol Biol Phys. 2002; 54:1524-1531.

32. Chatal JF, Davodeau F, Cherel M, Barbet J. Different ways to improve the clinical effectiveness of radioimmunotherapy in solid tumors. J Cancer Res Ther. 2009;5:S36-S40. 